\title{
Energy Cooperation in Energy Harvesting Wireless Communications
}

\author{
Berk Gurakan $^{1}$, Omur Ozel ${ }^{1}$, Jing Yang ${ }^{2}$, and Sennur Ulukus ${ }^{1}$ \\ ${ }^{1}$ Department of Electrical and Computer Engineering, University of Maryland, College Park, MD 20742 \\ ${ }^{2}$ Department of Electrical and Computer Engineering, University of Wisconsin-Madison, Madison, WI 53706
}

\begin{abstract}
We consider a simple multi-hop communication scenario composed of a source node, a relay node and a destination node where the source and the relay can harvest energy from the nature. Energy required for communication arrives (is harvested) at the transmitter and an unlimited battery stores it before being consumed for transmission. In addition, the source can assist the relay by transferring a portion of its energy to the relay through a separate energy transfer unit. We address this energy cooperation between the source and the relay in a deterministic setting. Assuming that the source and the relay nodes are informed of the energy arrivals in advance, we find jointly optimal offline energy management policies for the source and the relay that maximize the end-to-end throughput. We show that this problem is a convex problem. In order to gain insight about the structure of the solution, we consider specific scenarios. In particular, we show that if the relay energy profile is higher at the beginning and lower at the end with only one intersection, then matching the power sequences of the source and the relay slot-by-slot is optimal. We also consider the case when the energy of the source is available at the beginning and show that transferring energy in the first slot is optimal.
\end{abstract}

\section{INTRODUCTION}

User cooperation, achieved by means of relays, is known to enhance the system throughput in wireless communications [1]. In the classical setting of cooperation, relays help users in the transmission of their data by exploiting the broadcast nature of the wireless network. In this work, we anticipate an energy harvesting network where the nodes can share their energy as well as their information. We investigate an additional dimension of cooperation which involves the sharing of energy resources and can be described as energy cooperation.

We consider a simple multi-hop wireless communication system composed of a source node, a relay node and a destination node where the source and the relay nodes can harvest energy from the nature through various different sources, such as solar cells, vibration absorption devices, water mills, thermoelectric generators, etc. In particular, these nodes maintain the energy required for data transmission from the harvested energy after it is buffered in a rechargeable battery. Moreover, we assume that the source node can transfer a portion of its energy to the relay node as in Fig. 1 through a separate wireless energy transfer unit subject to an attenuation factor and a delay. We will address optimization of the energy

This work was supported by NSF Grants CCF 07-29127, CNS 09-64632, CCF 09-64645, CCF 10-18185 and CNS 11-47811.

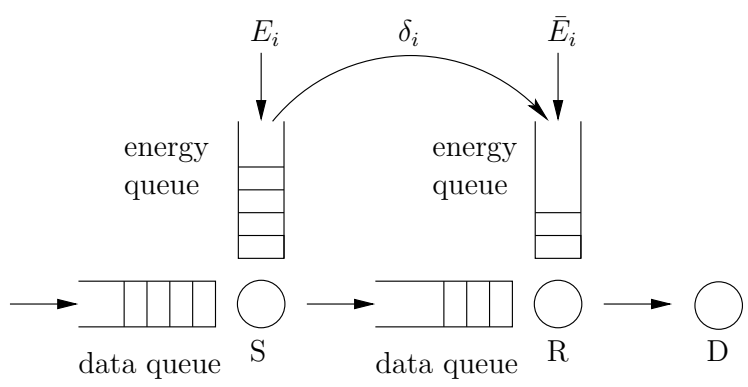

Fig. 1. Two-hop communication network with energy harvesting source and relay nodes and energy transfer from the source to the relay.

management policies of the source and the relay in this multi-hop communication scenario with energy harvesting and wireless energy transfer.

Offline throughput maximization for an energy harvesting transmitter has been recently analyzed by many researchers [2]-[12]. In [2]-[4], the problem of throughput maximization by a deadline is solved for an energy harvesting transmitter in a static channel. In [5]-[10], the throughput maximization problem and its solution are extended to fading, broadcast and multiple access channels. In [11], [12], end-to-end throughput maximization problem is solved in a two-hop cooperative network where jointly optimal policy for the source and the relay turns out to be separable: The source performs singleuser throughput maximization subject to its own energy arrival profile without regard to the relay energy profile and the relay adapts its transmission policy according to the data flow coming from the source as well as its own energy arrival profile. Moreover, this separable solution is not unique and thus there may be other optimal solutions that spend less energy at the source [11]. Unlike the problem in [11], [12], in our problem where energy cooperation is possible, the source is forced to be energy efficient so that it can further increase the throughput by sending some of its energy to the relay.

Another related line of research in wireless communications with energy harvesting nodes has been presented in [13]-[15]. In [13], capacity-energy function in simultaneous information and energy transfer is introduced and in [14] the tradeoff between the information rate and power transfer in a practical frequency selective wireless system is investigated. In [15], the tradeoff between energy and information transfer is characterized in a MIMO broadcast system. While the energy 
transfer in [13]-[15] is based on the RF technology which may be undesirable due to its inefficiency, there are other methods of wireless energy transfer based on magnetic coils that are known to be more efficient [16], [17].

Relying on the possibility of efficient energy transfer [16], [17], in this paper, we consider a two-hop communication network with energy harvesting nodes where the source assists the relay by transferring some of its energy. We extend the works in [11], [12] and formulate the end-to-end offline throughput maximization problem with wireless energy transfer subject to energy causality at both nodes and data causality at the relay node. We show that this problem is a convex optimization problem. In order to gain insight about the structure of the solution, we consider specific scenarios. If the relay energy profile is higher at the beginning and lower at the end with crossing only once, we show that slot-by-slot matching the power sequences of the source and the relay is optimal. Moreover, when the energy of the source is available at the beginning, transferring energy in the first slot is optimal. Our work reveals further relations between the problem of data transmission with an energy harvesting transmitter in [2]-[4] and the problem of energy minimal data transmission in [18], [19] under delay constraints.

\section{SYSTEM MODEL}

We consider a two-hop network consisting of a source node, a relay node and a destination node as shown in Fig. 1. The two queues at the source and the destination nodes are the data and energy queues. The energies that arrive at the source and the destination nodes are saved in the corresponding energy queues. The data queue of the source always carries some data packets to be delivered to the destination. The data packets sent from the source cause a depletion of the energy from the source energy queue and an increase in the relay data queue. Then, they are served out of this queue with a cost of energy depletion from the relay energy queue. We assume that the data and energy buffers are unlimited. In addition, energy expenditure is only due to data transmission; any other energy costs, e.g., processing, is not considered. There is a separate wireless energy transfer unit from the source to the relay and therefore, the source may wish to send a portion of its energy to the relay so that the relay can forward more data.

We assume additive white Gaussian noise (AWGN) channels from the source to the relay and from the relay to the destination. The received signals $y_{r}$ and $y_{d}$ at the relay and the destination, respectively, are given by $y_{r}=\sqrt{h_{s}} x_{s}+n_{s}$ and $y_{d}=\sqrt{h_{r}} x_{r}+n_{r}$, where $h_{s}$ and $h_{r}$ are the fading coefficients for the source-to-relay channel and the relay-to-destination channel, respectively. $n_{s}$ and $n_{r}$ are Gaussian noises each with zero-mean and unit-variance. We assume that $h_{s}=h_{r}=1$ without loss of generality as otherwise the energy arrivals can be properly scaled. The relay operates in full-duplex mode.

Time is slotted and there are a total of $T$ equal length slots. Without loss of generality, we assume that the length of each slot is unity. At times $t=1, \ldots, T$, the source harvests energy with amounts $E_{1}, E_{2}, \ldots, E_{T}$ and the relay harvests energy

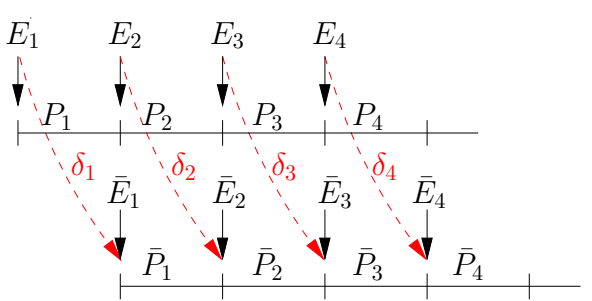

Fig. 2. Slotted system model: the queues of the relay is updated with one slot delay with respect to the queues of the source.

with amounts $\bar{E}_{1}, \bar{E}_{2}, \ldots, \bar{E}_{T}$. We assume an energy transfer efficiency of $\alpha$, where $0 \leq \alpha \leq 1$. This means that when the source transfers $\delta_{i}$ amount of energy to the relay through the wireless energy transfer unit, $\alpha \delta_{i}$ amount of energy enters the energy queue of the relay in the next slot. Similarly, the source uses $P_{i}$ power for data transmission and the data queue of the relay is increased by $\frac{1}{2} \log \left(1+P_{i}\right)$ bits in the next slot. Therefore, the source and relay queues are updated with one slot delay as shown in Fig. 2. Power policy of the source is the sequence $P_{i}$ and $\delta_{i}$; power policy of the relay is $\bar{P}_{i}$.

As the energy that has not arrived yet cannot be used for data transmission or energy transfer, the power policies of the source and the relay are constrained by the causality of energy in time. In particular, energy causality constraints are given as

$$
\begin{aligned}
& \sum_{i=1}^{k} P_{i} \leq \sum_{i=1}^{k}\left(E_{i}-\delta_{i}\right), \quad k=1, \ldots, T \\
& \sum_{i=1}^{k} \bar{P}_{i} \leq \sum_{i=1}^{k}\left(\bar{E}_{i}+\alpha \delta_{i}\right), \quad k=1, \ldots, T
\end{aligned}
$$

Moreover, the relay transmits data that arrives from the source. Therefore, the power policies of the source and the relay satisfy the following data causality constraints at the relay:

$$
\sum_{i=1}^{k} \frac{1}{2} \log \left(1+\bar{P}_{i}\right) \leq \sum_{i=1}^{k} \frac{1}{2} \log \left(1+P_{i}\right), \quad k=1, \ldots, T
$$

The optimal offline end-to-end throughput maximization problem with energy transfer subject to energy causality at both nodes and data causality at the relay node is:

$$
\begin{aligned}
\max _{\bar{P}_{i}, P_{i}, \delta_{i}} & \sum_{i=1}^{T} \frac{1}{2} \log \left(1+\bar{P}_{i}\right) \\
\text { s.t. } & \sum_{i=1}^{k} \frac{1}{2} \log \left(1+\bar{P}_{i}\right) \leq \sum_{i=1}^{k} \frac{1}{2} \log \left(1+P_{i}\right), \quad \forall k \\
& \sum_{i=1}^{k} P_{i} \leq \sum_{i=1}^{k}\left(E_{i}-\delta_{i}\right), \quad \forall k \\
& \sum_{i=1}^{k} \bar{P}_{i} \leq \sum_{i=1}^{k}\left(\bar{E}_{i}+\alpha \delta_{i}\right), \quad \forall k \\
& \sum_{i=1}^{k} \delta_{i} \leq \sum_{i=1}^{k} E_{i}, \quad \forall k
\end{aligned}
$$


We next show that the problem in (4) is a convex optimization problem. We use the change of variables from $\bar{P}_{i}, P_{i}, \delta_{i}$ to $\bar{r}_{i}=\frac{1}{2} \log \left(1+\bar{P}_{i}\right), r_{i}=\frac{1}{2} \log \left(1+P_{i}\right), \delta_{i}$. Then, the problem becomes:

$$
\begin{aligned}
\max _{\bar{r}_{i}, r_{i}, \delta_{i}} & \sum_{i=1}^{T} \bar{r}_{i} \\
\text { s.t. } & \sum_{i=1}^{k} \bar{r}_{i} \leq \sum_{i=1}^{k} r_{i}, \quad \forall k \\
& \sum_{i=1}^{k}\left(2^{2 r_{i}}-1\right) \leq \sum_{i=1}^{k}\left(E_{i}-\delta_{i}\right), \quad \forall k \\
& \sum_{i=1}^{k}\left(2^{2 \bar{r}_{i}}-1\right) \leq \sum_{i=1}^{k}\left(\bar{E}_{i}+\alpha \delta_{i}\right), \quad \forall k \\
& \sum_{i=1}^{k} \delta_{i} \leq \sum_{i=1}^{k} E_{i}, \quad \forall k
\end{aligned}
$$

The problem in (5) is a convex optimization problem as the objective function is concave and the feasible set is a convex set [20]. Therefore, (5) can be solved using standard techniques [20]. In particular, the Lagrangian function for the problem in (4) is:

$$
\begin{aligned}
\mathcal{L}= & -\sum_{i=1}^{T} \log \left(1+\bar{P}_{i}\right) \\
& +\sum_{k=1}^{T} \lambda_{k}\left(\sum_{i=1}^{k} \log \left(1+\bar{P}_{i}\right)-\sum_{i=1}^{k} \log \left(1+P_{i}\right)\right) \\
& +\sum_{k=1}^{T} \mu_{k}\left(\sum_{i=1}^{k} P_{i}-\left(E_{i}-\delta_{i}\right)\right) \\
& +\sum_{k=1}^{T} \eta_{k}\left(\sum_{i=1}^{k} \bar{P}_{i}-\left(\bar{E}_{i}+\alpha \delta_{i}\right)\right)-\sum_{k=1}^{T} \rho_{k} \delta_{k}
\end{aligned}
$$

The Lagrange multiplier $\rho_{k}$ is due to the constraint that $\delta_{k} \geq 0$. Note that the same constraints apply for $P_{i}$ and $\bar{P}_{i}$; however, $P_{i}$ and $\bar{P}_{i}$ are always nonzero in the optimal policy, therefore we exclude them. Similarly, we exclude the constraints $\sum_{i=1}^{k} \delta_{i} \leq \sum_{i=1}^{k} E_{i}$ in the Lagrangian function as these constraints can never be satisfied with equality in the optimal policy ${ }^{1}$. The KKT conditions for this problem are:

$$
\begin{array}{r}
\frac{-1+\sum_{k=i}^{T} \lambda_{k}}{1+\bar{P}_{i}}+\sum_{k=i}^{T} \eta_{k}=0, \quad i=1, \ldots, T \\
\frac{-\sum_{k=i}^{T} \lambda_{k}}{1+P_{i}}+\sum_{k=i}^{T} \mu_{k}=0, \quad i=1, \ldots, T \\
\sum_{k=i}^{T} \mu_{k}-\alpha \sum_{k=i}^{T} \eta_{k}-\rho_{i}=0, \quad i=1, \ldots, T
\end{array}
$$

\footnotetext{
${ }^{1}$ If the source transfers all of its arrived energy at some slot, the relay can have no data to send at that slot. Allocating a small portion of energy for source transmission strictly increases the throughput in this case.
}

with the additional complementary slackness conditions as:

$$
\begin{array}{rlrl}
\lambda_{k}\left(\sum_{i=1}^{k} \log \left(1+\bar{P}_{i}\right)-\sum_{i=1}^{k} \log \left(1+P_{i}\right)\right) & =0, & \forall k \\
\mu_{k}\left(\sum_{i=1}^{k} P_{i}-\left(E_{i}-\delta_{i}\right)\right) & =0, & & \forall k \\
\eta_{k}\left(\sum_{i=1}^{k} \bar{P}_{i}-\left(\bar{E}_{i}+\alpha \delta_{i}\right)\right) & =0, & & \forall k \\
\rho_{k} \delta_{k} & =0, & & \forall k
\end{array}
$$

From (7), (8) and (9) we get:

$$
\begin{aligned}
\bar{P}_{i} & =\frac{1-\sum_{k=i}^{T} \lambda_{k}}{\sum_{k=i}^{T} \eta_{k}}-1, \quad i=1, \ldots, T \\
P_{i} & =\frac{\sum_{k=i}^{T} \lambda_{k}}{\sum_{k=i}^{T} \mu_{k}}-1, \quad i=1, \ldots, T \\
\rho_{i} & =\sum_{k=i}^{T} \mu_{k}-\alpha \sum_{k=i}^{T} \eta_{k} \quad i=1, \ldots, T
\end{aligned}
$$

Next, we will obtain necessary optimality conditions for (4).

\section{Necessary Optimality CONDitions}

The first necessary optimality condition for (4) is that the source has to send as many bits as the relay can send and the relay has to finish up all the data in its data buffer. In other words, in the optimal policy, no data is left in the data queue of the relay at the end.

Lemma 1 The optimal power sequences $P_{i}^{*}, \bar{P}_{i}^{*}$ must satisfy the constraint $\sum_{i=1}^{T} \frac{1}{2} \log \left(1+\bar{P}_{i}^{*}\right)=\sum_{i=1}^{T} \frac{1}{2} \log \left(1+P_{i}^{*}\right)$.

Proof: Suppose the stated constraint is satisfied with strict inequality. Then, we can increase some $\delta_{i}^{*}$, increase $\bar{P}_{i}$ and decrease $P_{i}$ without violating the energy constraints and improve the overall throughput which contradicts the fact that $\bar{P}_{i}^{*}, P_{i}^{*}$ and $\delta_{i}^{*}$ are optimal.

We note that if the relay energy profile is sufficient to forward all the bits in the optimal source data stream with respect to the source energy profile, that is, if the separable policy in [11], [12] yields a policy that satisfies the necessary condition in Lemma 1, then it is the optimal solution for (4) and energy transfer is not needed. In fact, any energy transfer from source to relay causes a strict suboptimality in this case.

The second observation about the optimal policy is that the source has to exhaust the energies that have been harvested throughout the communication session either for data transmission or in the form of energy transfer.

Lemma 2 The optimal power profiles $P_{i}^{*}, \bar{P}_{i}^{*}$ and energy transfers $\delta_{i}^{*}$ must satisfy $\sum_{i=1}^{T} P_{i}^{*}=\sum_{i=1}^{T}\left(E_{i}-\delta_{i}^{*}\right)$.

Proof: Suppose this constraint is satisfied with strict inequality. Then, we can increase some $\delta_{i}^{*}$ and then increase $P_{i}^{*}$ and $\bar{P}_{i}^{*}$ to achieve a larger throughput and satisfy the constraints of (4). This contradicts the optimality of $P_{i}^{*}, \bar{P}_{i}^{*}, \delta_{i}^{*}$. 
Another necessary condition satisfied by the optimal policy $P_{i}^{*}, \bar{P}_{i}^{*}$ is energy minimality. Consider the energy minimization problem subject to data causality [18], [19] where $e_{i}$ are energy allocated to packets and $s \in[1: T]$ is a deadline:

$$
\begin{array}{ll}
\min _{e_{i}} & \sum_{i=1}^{s} e_{i} \\
\text { s.t. } & \sum_{i=1}^{k} \frac{1}{2} \log \left(1+e_{i}\right) \leq \sum_{i=1}^{k} \frac{1}{2} \log \left(1+P_{i}^{*}\right), k \neq s \\
& \sum_{i=1}^{s} \frac{1}{2} \log \left(1+e_{i}\right)=\sum_{i=1}^{s} \frac{1}{2} \log \left(1+P_{i}^{*}\right)
\end{array}
$$

The optimal solution for (17) is $e_{i}^{*}=P_{i}^{*}$ for any $s \in[1: T]$. That is, the power consumption profile of $P_{i}^{*}$ in any interval $[1: s]$ has to be the minimum energy consuming profile that has the data profile of $P_{i}^{*}$ in $[1: s]$. The energy minimality condition is also true for $\bar{P}_{i}^{*}$. A corollary of the energy minimality is that if $\sum_{i=1}^{T} \bar{E}_{i}<\sum_{i=1}^{T} E_{i}$, i.e., if the total relay energy is less than the total source energy, then the relay cannot forward the source data stream with its own energy. In this case, $\exists i, \delta_{i}^{*} \neq 0$, i.e., some energy transfer is needed.

Finally, we observe that if there is a nonzero energy transfer from the source to the relay, then the relay has to exhaust all of its energy in the optimal policy.

Lemma 3 For the optimal power sequences $P_{i}^{*}, \bar{P}_{i}^{*}$ and energy transfer sequence $\delta_{i}^{*}$, if $\delta_{i}^{*} \neq 0$ for some $i$, then $\sum_{i=1}^{T} \bar{P}_{i}^{*}=\sum_{i=1}^{T}\left(\bar{E}_{i}+\alpha \delta_{i}^{*}\right)$.

Proof: Suppose this constraint is satisfied with strict inequality. Using a similar argument as in Lemma 2, we can decrease $\delta_{i}^{*}$ and increase $\bar{P}_{i}^{*}$ to achieve a larger throughput and satisfy the constraints of problem (4). This contradicts the optimality of $P_{i}^{*}, \bar{P}_{i}^{*}, \delta_{i}^{*}$.

Necessary conditions in Lemmas 1 through 3 do not provide detailed structural properties of the optimal policy for an algorithmic solution. In the next section, we consider specialized scenarios to gain insight on the jointly optimal policy.

\section{SPECIFIC SCEnARIOS}

In this section, we examine specific scenarios corresponding to practically interesting cases such as the cases of the source or the relay with a fixed energy battery. Furthermore, they provide useful insights on how to optimally allocate power and transfer energy simultaneously.

\section{A. Relay Energy Higher at the Beginning Lower at the End}

We consider the scenario where the relay energy arrival profile is higher at the beginning, intersects the energy arrival profile of the source only once and remains lower till the end of the communication as shown in Fig. 3. In particular, we assume that there exists $\tilde{i} \in[0: T]$ such that $\sum_{k=1}^{i} \bar{E}_{k} \geq$ $\sum_{k=1}^{i} E_{k}$ for all $i \in[1: \tilde{i}]$ and $\sum_{k=1}^{i} \bar{E}_{k} \leq \sum_{k=1}^{i} E_{k}$ for all $i \in[\tilde{i}+1: T]$. In Fig. 3, $\tilde{i}=3$. We note that this case also covers the setting where the relay is non energy harvesting

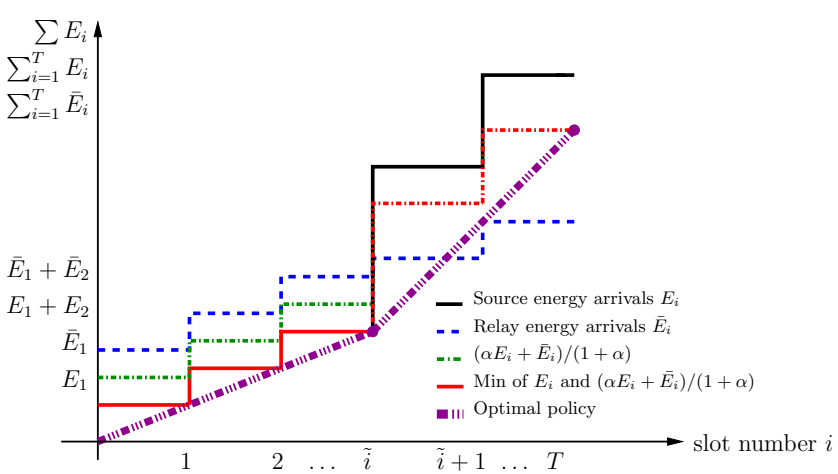

Fig. 3. Optimal power sequence and energy transfer when the relay energy profile is higher at the beginning and lower at the end with crossing once.

and only the source harvests energy during the communication session. We propose the following solution: Form a new energy arrival profile $\min \left\{\sum_{k=1}^{i} \frac{E_{k}+\alpha E_{k}}{\alpha+1}, \sum_{k=1}^{i} E_{k}\right\}$ as in Fig. 3 and maximize throughput with respect to this profile. In particular, use $\sum_{k=1}^{i} E_{k}$ for $i \in[1: \tilde{i}]$ and $\sum_{k=1}^{i} \frac{\widehat{E}_{k}+\alpha E_{k}}{\alpha+1}$ for $i \in[\tilde{i}+1: T]$; energy transfer is performed only at slots $[\tilde{i}+1: T]$. The resulting power sequence is matched for the source and the relay. More specifically, we propose

$P_{i}^{*}=\bar{P}_{i}^{*}=\frac{\min \left\{\frac{\sum_{j=n_{i-1}}^{n_{i}} \bar{E}_{j}+\alpha E_{j}}{\alpha+1}, \sum_{j=n_{i-1}}^{n_{i}} E_{j}\right\}}{n_{i}-n_{i-1}}$
$n_{i}=\arg \min _{n_{i-1} \leq k \leq T}\left\{\frac{\min \left\{\sum_{j=n_{i-1}}^{k} \frac{\bar{E}_{j}+\alpha E_{j}}{\alpha+1}, \sum_{j=n_{i-1}}^{k} E_{j}\right\}}{k-n_{i-1}}\right\}$

We next show that there exist $\lambda_{i}, \mu_{i}, \eta_{i}, \rho_{i} \geq 0$ that satisfy (7)(13) and yield the solution in (19) via (14)-(16). In particular, $\rho_{i}=0$ and $\eta_{i}=\frac{\mu_{i}}{\alpha}$ for $i \in[\tilde{i}+1: T]$. Since $\alpha \sum_{k=i}^{T} \eta_{k}=$ $\sum_{k=i}^{T} \mu_{k}$ for all $i \in[\tilde{i}+1: T]$, we have from (14) and (15)

$$
\bar{P}_{i}^{*}+\alpha P_{i}^{*}=\frac{1}{\sum_{k=i}^{T} \eta_{k}}-(1+\alpha), \quad i \in[\tilde{i}+1: T]
$$

Hence, $\bar{P}_{i}^{*}=\frac{1}{(1+\alpha) \sum_{k=i}^{T} \eta_{k}}-1$, which implies that $\lambda_{T}=\frac{1}{2}$ and $\lambda_{i}=0$ for $i \in[\tilde{i}+1: T-1]$. Moreover, $\eta_{i}=\frac{\mu_{i}}{\alpha}>0$ whenever $\sum_{k=1}^{i} \frac{\bar{E}_{k}+\alpha E_{k}}{\alpha+1}$ is active for some $i \in[\tilde{i}+1: T]$. As in [6], [7], we can show that such $\eta_{i}=\frac{\mu_{i}}{\alpha}$ that yield the power sequence in (19) are uniquely found for $i \in[\tilde{i}+1: T]$.

It remains to find the Lagrange multipliers for $i \in[1: \tilde{i}]$. We observe that $\eta_{i}=0$ and $\rho_{i}=\sum_{k=i}^{\tilde{i}} \mu_{k}$ for $i \in[1: \tilde{i}]$. That is, the relay power constraint is not active in the first $i$ slots. This is due to the necessary energy minimality condition on $P_{i}^{*}$ and $\bar{P}_{i}^{*}$ mentioned in Section III, which forces $\sum_{k=1}^{i} \bar{P}_{k}^{*}<$ $\sum_{k=1}^{i} \bar{E}_{k}, i \in[1: \tilde{i}]$. Hence, $\sum_{k=i}^{T} \eta_{k}=\sum_{k=\tilde{i}+1}^{T} \eta_{k}$, i.e., constant for all $i \in[1: \tilde{i}]$. As $\bar{P}_{i}^{*} \leq \bar{P}_{\tilde{i}+1}^{*}$, we specify $\lambda_{i} \in$ $\left[0, \frac{1}{2}\right]$ recursively, with $\lambda_{i}>0$ only when $\sum_{k=1}^{i} E_{k}$ constraint is active, as: $\lambda_{i}=1-\bar{P}_{i}^{*} \sum_{k=\tilde{i}+1}^{T} \eta_{k}-\sum_{k=i+1}^{T} \lambda_{k}$.

Moreover, $\mu_{i}>0$ for slots where $\sum_{k=1}^{i} E_{k}$ constraint is active and $\mu_{i}=\frac{\sum_{k=i}^{T} \lambda_{k}}{P_{i}^{*}}-\sum_{k=i+1}^{T} \mu_{k}$. Note that the optimal source and relay power sequences are unique but there may be 
infinitely many $\delta_{i}^{*}$ that allow the same optimal power levels.

A particular case covered is when only the source has energy replenishments and the relay has all its energy initially available, i.e., $\bar{E}_{1}>0$ and $\bar{E}_{i}=0, i>1$. If $\bar{E}_{1}>\sum_{i=1}^{T} E_{i}$, the relay can forward all the bits sent from the source and the optimal policy is trivial. If $\bar{E}_{1}<\sum_{i=1}^{T} E_{i}$, the optimal policy is obtained by forming a common energy profile via energy transfer and matching the power and rate sequences. Another special case is when $\tilde{i}=0$, i.e., when $\bar{E}_{i}<E_{i}$ for all $i$. In this case, $\min \left\{\sum_{k=1}^{i} \frac{\bar{E}_{k}+\alpha E_{k}}{\alpha+1}, \sum_{k=1}^{i} E_{k}\right\}=\sum_{k=1}^{i} \frac{\alpha \bar{E}_{k}+E_{k}}{\alpha+1}$ for all $i$ and matching the relay and source power sequences is optimal with $\delta_{i}^{*}=E_{i}-\frac{\bar{E}_{i}+\alpha E_{i}}{\alpha+1}$. When $\tilde{i}=T$, we have $\bar{E}_{i}>E_{i}, \forall i$. The source optimizes the throughput according to $\left\{E_{i}\right\}_{i=1}^{T}$ and the relay power is matched with the source.

\section{B. Source Energy Available at the Beginning}

In this section, we consider the scenario where the source has all the available energy in the beginning (i.e., $E_{1}>0$ only) and the relay harvests energy throughout the communication. Let the relay energy profile not be satisfactory to forward the optimal source data stream which has constant rate $\frac{E_{1}}{T}$. Assume $\delta_{i} \neq 0$ for some $i$. Since the source is not harvesting energy, the total energy of the source will then be $E_{1}-\delta_{i}$ yielding an optimal transmission power of $\frac{E_{1}-\delta_{i}}{T}$. Hence, the throughput of the source is independent of the slot index $i$ the energy is transferred. However, transferring the energy at slot $j<i$ can only increase the powers after that slot; therefore, energy transfer has to be performed as early as possible, i.e., at the first slot. Hence, the jointly optimal policy is $\delta_{1}^{*} \neq 0$ and $\delta_{i}^{*}=0$ for the remaining slots as shown in Fig. 4. Note that the power sequences of the source and the relay are not matched. $\delta_{1}^{*}$ is found by solving a fixed point equation:

$$
f\left(\bar{E}_{1}+\delta_{1}^{*}, \bar{E}_{2}, \ldots, \bar{E}_{T}\right)=\frac{T}{2} \log \left(1+\frac{E_{1}-\delta_{1}^{*}}{T}\right)
$$

where $f\left(\bar{E}_{1}, \bar{E}_{2}, \ldots, \bar{E}_{T}\right)$ is the maximum number of bits corresponding to the energy arrival sequence $\bar{E}_{1}, \bar{E}_{2}, \ldots, \bar{E}_{T}$.

\section{CONCLUSIONS}

In this paper, we considered a two-hop communication network with energy harvesting rechargeable nodes where the source node and the relay node are in energy as well as signal cooperation: the relay assists the source node by forwarding the data to the destination; the source assists the relay by transferring a portion of its energy to the relay. We formulated the offline end-to-end throughput maximization problem with energy transfer from the source to the relay subject to energy causality at both nodes and data causality at the relay node and we showed that it is a convex optimization problem. We studied specific cases in detail, e.g., when the relay energy profile is initially higher and then lower with one crossing, as well as the case when the source energy is constant.

\section{REFERENCES}

[1] A. Sendonaris, E. Erkip, and B. Aazhang, "User cooperation diversity. Part I. System description,” IEEE Trans. Comm., vol. 51, pp. 1927 1938, November 2003.

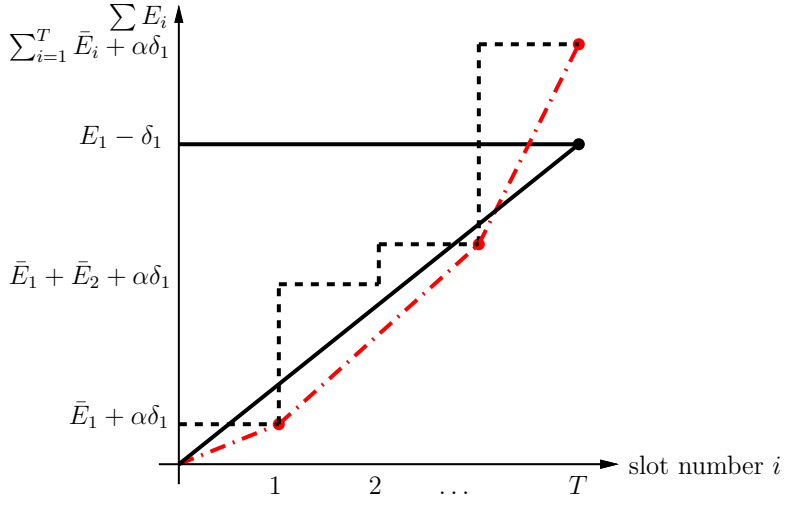

Fig. 4. Power allocation profiles for the source and relay after energy transfer.

[2] J. Yang and S. Ulukus, "Transmission completion time minimization in an energy harvesting system," in CISS, March 2010.

[3] J. Yang and S. Ulukus, "Optimal packet scheduling in an energy harvesting communication system," IEEE Trans. Comm., vol. 60, pp. 220-230, January 2012.

[4] K. Tutuncuoglu and A. Yener, "Optimum transmission policies for battery limited energy harvesting nodes," IEEE Trans. Wireless Comm., vol. 11, pp. 1180-1189, March 2012.

[5] C. Ho and R. Zhang, "Optimal energy allocation for wireless communications powered by energy harvesters," in IEEE ISIT, June 2010.

[6] O. Ozel, K. Tutuncuoglu, J. Yang, S. Ulukus, and A. Yener, "Transmission with energy harvesting nodes in fading wireless channels: Optimal policies," IEEE Jour. on Selected Areas in Commun., vol. 29, pp. 1732 1743, September 2011.

[7] J. Yang, O. Ozel, and S. Ulukus, "Broadcasting with an energy harvesting rechargeable transmitter," IEEE Trans. Wireless Comm., vol. 11, pp. 571-583, February 2012.

[8] M. A. Antepli, E. Uysal-Biyikoglu, and H. Erkal, "Optimal packet scheduling on an energy harvesting broadcast link," IEEE Jour. on Selected Areas in Commun., vol. 29, pp. 1721-1731, September 2011.

[9] J. Yang and S. Ulukus, "Optimal packet scheduling in a multiple access channel with rechargeable nodes," in IEEE ICC, June 2011.

[10] J. Yang and S. Ulukus, "Optimal packet scheduling in a multiple access channel with rechargeable nodes," Journal of Communications and Networks, vol. 14, pp. 140-150, April 2012.

[11] C. Huang, R. Zhang, and S. Cui, "Throughput maximization for the Gaussian relay channel with energy harvesting constraints," IEEE Jour. on Selected Areas in Commun., submitted, September 2011. Also available at [arXiv:1109.0724].

[12] D. Gunduz and B. Devillers, "Two-hop communication with energy harvesting," in IEEE CAMSAP, December 2011.

[13] L. Varshney, "Transporting information and energy simultaneously," in IEEE ISIT, July 2008.

[14] P. Grover and A. Sahai, "Shannon meets Tesla: Wireless information and power transfer," in IEEE ISIT, July 2010.

[15] R. Zhang and C. K. Ho, "MIMO broadcasting for simultaneous wireless information and power transfer," submitted, May 2011. Also available at [arXiv: 1105.4999].

[16] A. Kurs, A. Karalis, R. Moffatt, J. Joannopoulos, P. Fisher, and M. Soljacic, "Wireless power transfer via strongly coupled magnetic resonances," Science, vol. 317, pp. 83-86, Jul. 2007.

[17] A. K. J. Joannopoulos and M. Soljacic, "Efficient wireless non-radiative mid-range energy transfer," Annals of Physics, vol. 323, pp. 34 - 48, Jan. 2008

[18] E. Uysal-Biyikoglu, B. Prabhakar, and A. El Gamal, "Energy-efficient packet transmission over a wireless link," IEEE/ACM Trans. on Networking, vol. 10, pp. 487-499, August 2002.

[19] M. Zafer and E. Modiano, "A calculus approach to energy-efficient data transmission with quality-of-service constraints," IEEE/ACM Trans. Networking, vol. 17, pp. 898-911, June 2009.

[20] S. Boyd and L. Vandenberghe, Convex Optimization. United Kingdom: Cambridge University Press, 2004. 